\title{
Virtual Screening and Validation of Potential Lead Compound from the Malaria Box against Plasmodium Falciparum S7 and S19 Proteins
}

\section{Mwangi HN ${ }^{1}$, Onyango $\mathrm{S}^{2}$, Omosa $\mathrm{LK}^{2}$ and Mulaa $\mathrm{F}^{1 *}$}

${ }^{1}$ Department of Biochemistry, College of Health Sciences, University of Nairobi, Kenya ${ }^{2}$ Department of Chemistry, College of Physical and Applied Sciences, University of Nairobi, Kenya

*Corresponding author: Francis Mulaa, Department of Biochemistry, College of Health Sciences, Riverside Drive, Chiromo Campus, University of Nairobi, Nairobi, Kenya, Tel: +254733876968; Email: mulaafj@uonbi.ac.ke

\section{Research Article \\ Volume 2 Issue 2}

Received Date: February 17, 2018

Published Date: April 30, 2018

\section{Abstract}

Molecular docking is a computer based tool used in drug design, where predominant binding modes of a ligand with known three dimensional protein structures are predicted. This study addresses the possible ways of coming up with antimalarial drugs using computational chemistry methods. This approach which utilizes virtual screening is more advantageous as it is effective, affordable and less tedious as compared to the normal laboratory experimental biological assays and spectroscopic techniques for characterization of the compounds. Treatment of malaria has become complex due to the high rate of emergence of resistance exhibited by Plasmodium falciparum and mutations resulting from the parasite. To test the reliability of this model for future tests, compounds for this study were sourced from the Malaria Box containing compounds proven to have interesting activities against the malaria causing parasite, P. faliciparum. In the current study, three dimensional structures of twenty compounds fetched from the Malaria Box and of the ribosomal 40S proteins S7 and S19 were generated using structure predicting software. The potential of the selected compounds to dock and bind onto the ribosomal 40S proteins S7 and S19 proteins was accessed by calculating pose complex RMSD as well as its geometric shape complementarity score, approximate interface area, atomic contact energy, the $3 \mathrm{~d}$ transformation, 3 rotational angles and 3 translational parameters applied on the ligand molecule, to test authenticity of the docking experiments. The compounds that showed best pose were considered active lead compounds. Using this model three out of several compounds from the Malaria Box confirmed the results that were obtained using laboratory experiments as evidenced from the low energy pose of the complex. The objectives of the experiment were accomplished as the Plasmodium falciparum proteins S7 and S19 models were generated, validated and the docked structures of the two proteins with the 


\section{Open Access Journal of Pharmaceutical Research}

ligands generated. Through the research it was evident that the compounds in the malaria box library could be screened easily, quickly and perfectly to eliminate experimental false positives and negatives. These results could help expedite the process of bringing a more effective class of antimalarial drugs as the results show that they have activity against the proteins with further in vitro validation.

Keywords: Computation chemistry; proteins docking; malaria box; homology modeling; Plasmodium falciparu; RMSD

Abbrevations: PDB: Protein Data Bank; NCBI: National Centre for Biotechnology Information; ACE: atomic contact energy.

\section{Introduction}

Ribosome is the site for protein synthesis which are needed for cells to perform their cellular activities. Ribosomes are derived from complexes of rRNAs and proteins [1]. Ribosomes are contained in both prokaryotes and eukaryotes. Eukaryotic and prokaryotic ribosomes differ in their composition especially in the smaller subunit [2]

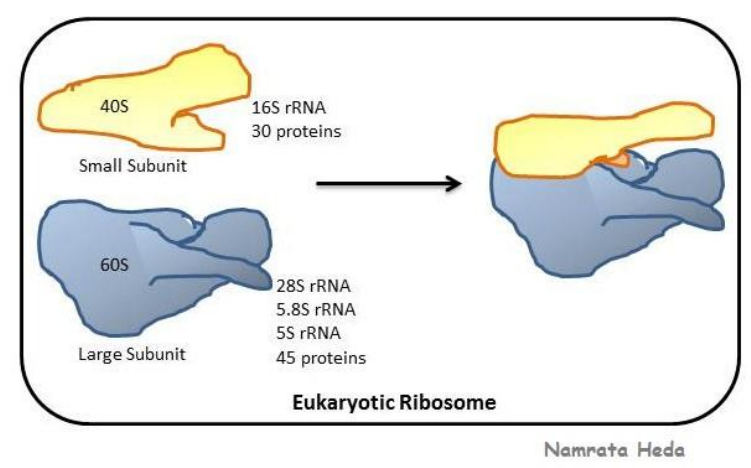

Figure 1: Structure of the Ribosome showing the two main components of the ribosome each made of the ribosomal RNA and proteins.

The 40S subunit comprises the decoding center which controls the complementarity of tRNA and mRNA in protein translation. The eukaryotic small ribosomal subunit (40S) plays a central role in this process [2]; it binds initiation factors that promote scanning of messenger RNAs and initiation of protein synthesis [3].

Ribosome has structural differences between organisms. It is these structural differences which make drugs be particular on the parasite ribosome and not the host's (human being) ribosome. Ribosome target is important because the cellular activity of the parasite will stop and the parasite will die.

Ribosomal S7 and S19 proteins are important in that they provide instructions for making other different ribosomal proteins, which are components of cellular structures [4]. Ribosomes are what then receive the cell's genetic signal to create proteins. They are also involved in the assembly or stability of ribosomes in general [5]. Besides carrying out ribosome's main activity of building new proteins, they are also involved in other activities such as participating in chemical signaling pathways within the cell, controlling the self-destruction of cells and regulating cell division [6].

People from all sectors of the world are being affected by Malaria. The parasite causing malaria is also becoming resistant to various drugs in the market. Malaria has economic effects in a country and the world at large. The economic effects include reduced population hence low labor supply leading to low capital [7].

Malaria if not controlled can lead many orphans resulting too many cases of school dropouts hence no needed skilled labor in the market. Malaria parasite usually mutate making the treatment of malaria very complex [8,9]. Plasmodium falciparum also develops resistance to some ant malarial drugs available. As a result of this, malaria should be controlled as fast as possible to reduce the number of deaths which usually occur [10].

\section{Methodology}

Vast advancements have been made in the fight against malaria. Various control strategies example development of ant malarial drugs has helped reduced the number of malaria-related deaths across the world. Positive results in the fight against malaria have been reported through 


\section{Open Access Journal of Pharmaceutical Research}

development and administration effective drugs, the combination of interventions which include timely diagnosis, household spraying with long-lasting insecticides and use of treated bed nets insecticide which bars mosquito from biting people hence causing malaria at night. Though this has helped there is need to provide newer intervenes against malaria that are more tailor made to completely eradicate the disease. The technology proposed in this project shows that this is posible to be done through computational chemistry effectively and efficiently.

With the advancement in technology, tools for structure-based drug discovery are proliferating rapidly. Models bounded by the Lock-and-Key and Induced-Fit theories for ligand binding can be generated.

Molecular modeling involves:

1. Template Identification and selection - Many templates will be generated and then the most suitable one identified. A suitable template will then be selected to generate the best model. The score between the template and that of the target sequence must be considered when selecting the template.

2. Template alignment and selection - In building a model, the modeling procedures depend on the structural similarities between the template and target residues defined by the alignment of the template and target sequences. After a template has been selected, an alignment is to be.

3. Model building, realignment and optimization - Once the template-target alignment is constructed, various methods can be employed to generate a 3D model for the target protein. The methods included, modeling through segment matching which is based on the approximated positions of the conserved atoms in the templates to generate the atoms' coordinates. MODELLER, which is used to generate 3D structures, extracts spatial restraints from homology-derived restraints brought by the distances and dihedral angles in the target sequence are extracted from its alignment with the template structures and stereo chemical restraints such as bond length and bond angle. The modeling is then optimized to ensure conceptual simplicity and energy minimization.
4. Model validation - The generated model after alignment is then validated by accurate checking to remove possible errors where its quality effectiveness will be evident from the similarity in sequence between the target and the template. Internal evaluation is done to check whether the model satisfied the restraints used in its calculation. It is then evaluated through assessment of the stereochemistry of the model which includes bond angles, dihedral angles, non-bonded atom-atom distances and bonds [11-15].

Molecular docking generally is the placing of a small molecule, ligand, into an active site in a receptor in such a way that there are optimal interactions with the receptor. There are two types of docking; 1. Lock and Key Docking in this case, both the internal geometry of the ligand and the receptor are kept fixed and are not interfered with during docking. 2. Induced fit - The ligand is kept flexible in this case and the energy for various conformations of the ligand fitting into the protein is calculated. This can be diagrammatically shown below:

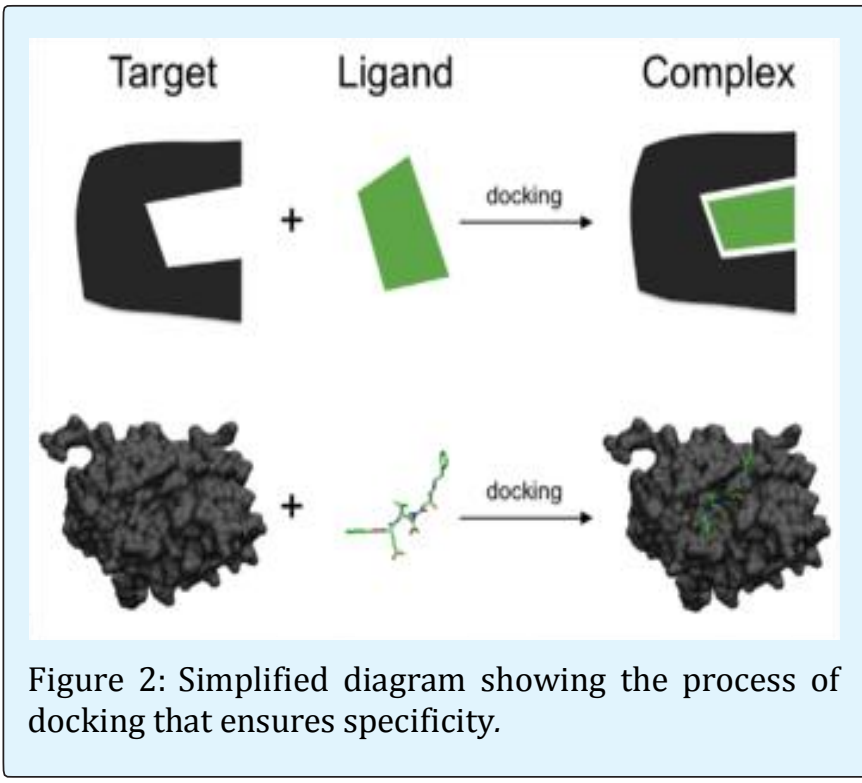

\section{Results and Discussion}

2 of the 20 selected compounds have been shown in the tables below: 


\begin{tabular}{|c|c|}
\hline IUPAC Name & 7-(pyridine-4-yloxy)isoquinolin-1-amine \\
\hline Accession Number & MMV026313 \\
\hline Structure & \\
\hline Molecular weight & Nc1nccc2ccc(Oc3ccncc3)cc12 \\
\hline Chemical Formula & $\mathrm{C}_{14} \mathrm{H}_{11} \mathrm{~N}_{3} \mathrm{O}$ \\
\hline SMILES & \\
\hline
\end{tabular}

Table 1: MMV Pathogen box compound MMV026550, with detail description of the IUPAC name, molecular weight, modeled secondary structure and its three dimensional structure, its chemical formulae and its smile format.

\begin{tabular}{|c|c|}
\hline IUPAC Name & 1-neopentyl-4,4-diphenyl-1H-imidazol-5(4H)-one \\
\hline Accession Number & \\
\hline Molecular weight & CC(C)(C)CN1C(=NC(C1=0)(c2ccccc2)c3cccc3) \\
\hline Chemical Formula & 321.42 \\
\hline SMILES & $\mathrm{C}_{20} \mathrm{H}_{23} \mathrm{~N}_{3} \mathrm{O}$ \\
\hline
\end{tabular}

Table 2: MMV Pathogen box compound MMV026313, with detail description of the IUPAC name, molecular weight, modelled secondary structure and its three dimensional structure, its chemical formulae and its smile format.

The figures below show the 3D orientations of the ribosomal proteins S7 and S19 respectively: 


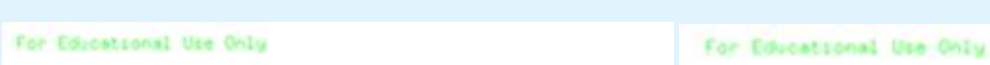
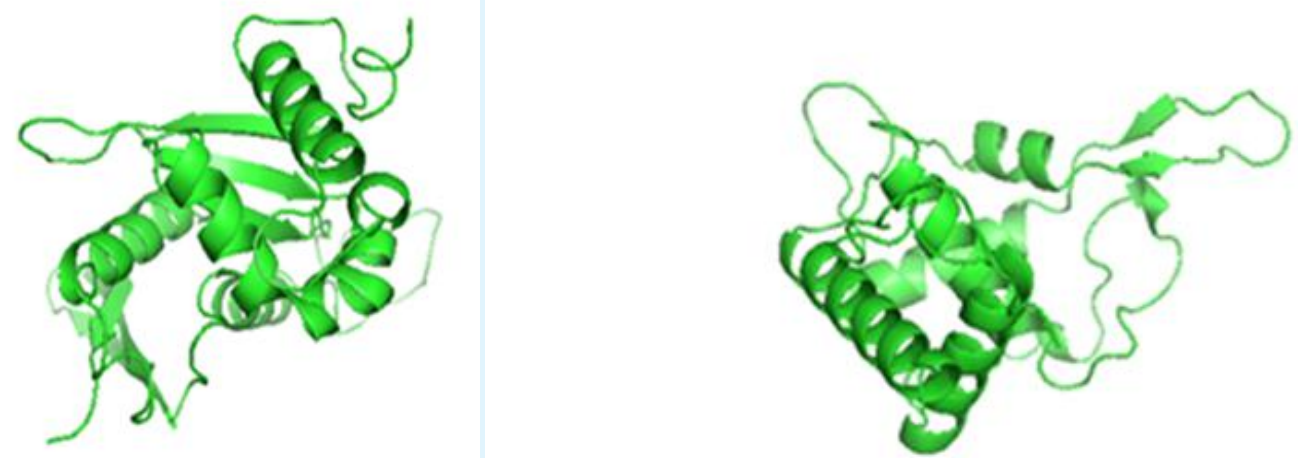

Figure 3: Three dimensional structure of Plasmodium falciparum 40S ribosomal Protein s7 on the left and s19 on the right.

The compounds were then docked to the ribosomal proteins above and the results obtained as follows; Protein S7 docked with 026MMV676269

\begin{tabular}{|c|c|c|c|c|}
\hline $\begin{array}{c}\text { Solution } \\
\text { No }\end{array}$ & Score & Area & ACE & Transformation \\
\hline 1 & 4404 & 520.00 & -35.43 & $\begin{array}{c}-1.40-0.55-2.86212 .17 \\
321.05321 .65\end{array}$ \\
\hline
\end{tabular}

Table 1: The best solution after docking with the geometric shape complementarity score of 4404, approximate interface area of the complex, atomic contact energy, the $3 \mathrm{~d}$ transformation, 3 rotational angles and 3 translational parameters applied on the ligand molecule.

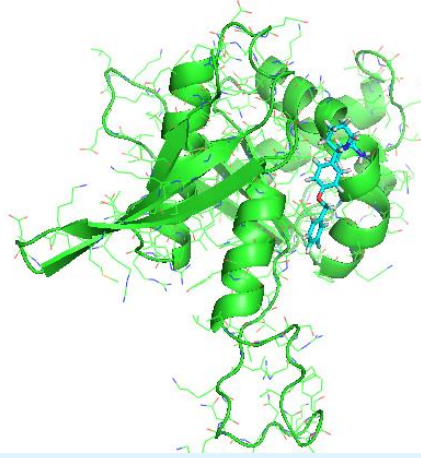

Figure 4: The predicted complex structure in PDB format of Plasmodium falciparum 40SProtein S7 docked with 026MMV676269.
Protein S7 docked with 027.MMV676270

\begin{tabular}{|c|c|c|c|c|}
\hline $\begin{array}{c}\text { Solution } \\
\text { No }\end{array}$ & Score & Area & ACE & Transformation \\
\hline 1 & 4790 & 585.60 & -44.94 & $\begin{array}{c}2.83-0.570 .46199 .78 \\
330.34334 .21\end{array}$ \\
\hline
\end{tabular}

Table 4: The best solution after docking with the geometric shape complementarity score of 4790, approximate interface area of the complex , atomic contact energy, the $3 \mathrm{~d}$ transformation, 3 rotational angles and 3 translational parameters applied on the ligand molecule.

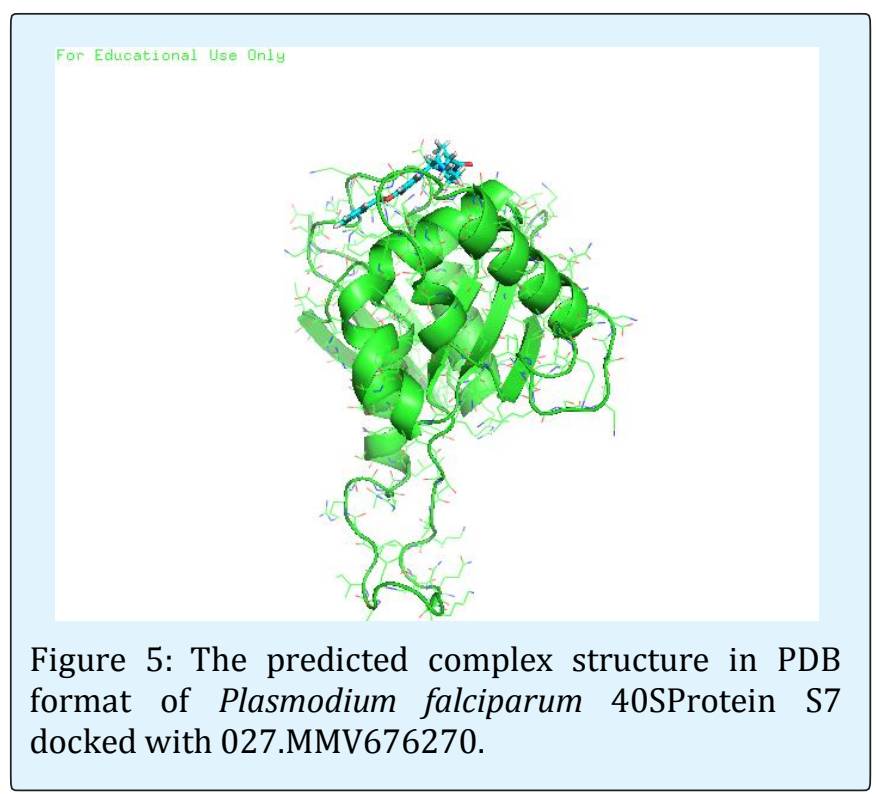

Mulaa F, et al. Virtual Screening and Validation of Potential Lead Compound from the 


\section{Open Access Journal of Pharmaceutical Research}

Protein S19 docked with compound 024.MMV026550.

\begin{tabular}{|c|c|c|c|c|}
\hline $\begin{array}{c}\text { Solution } \\
\text { No }\end{array}$ & Score & Area & ACE & Transformation \\
\hline 1 & 4176 & 455.70 & -101.28 & $\begin{array}{c}1.69-1.21-2.36117 .75 \\
214.28272 .60\end{array}$ \\
\hline
\end{tabular}

Table 5: The best solution after docking with the geometric shape complementarity score of 4176, approximate interface area of the complex , atomic contact energy, the $3 \mathrm{~d}$ transformation, 3 rotational angles and 3 translational parameters applied on the ligand molecule.

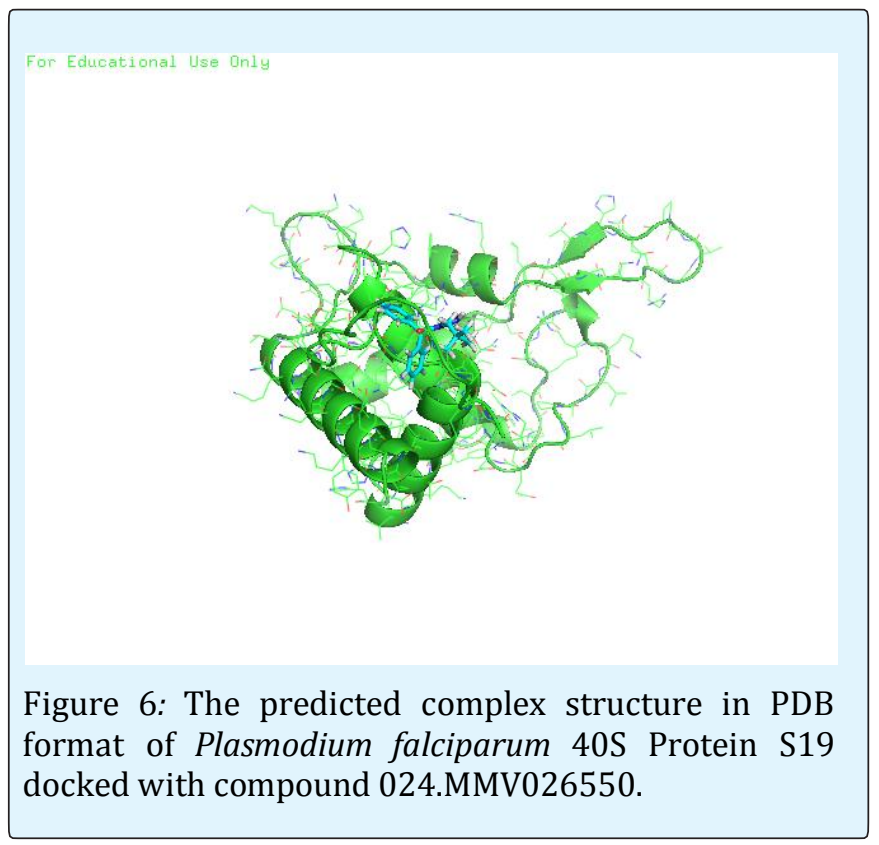

The 3D structures of the selected 20 compounds from Malaria Box were generated as well as their corresponding 2D structures. Their IUPAC names, Chemical formula, Molecular weight were also documented. These compounds from Malaria Box are compounds which have been isolated, characterized and tested in the wet lab assays and confirmed to have moderate to high antiplasmodial activities with $\mathrm{IC}_{50}$ values ranging from $1-10 \mathrm{mg} / \mathrm{ml}$. The two protein structures S7 and S19 were also generated using the Schrodinger software - Maestro. The sequences were obtained from Protein Data Bank (PDB) and National Centre for Biotechnology Information (NCBI) which is online databases. Screening for the 20 compounds against Plasmodium falciparum 40S proteins S7 and S19 were done and they were found to be good compounds against the parasite as they docked perfectly into the proteins structures with the high geometric shape score, good interface area and minimal atomic contact energy (ACE).

The above docked results are samples from the docking experiment. Best poses of the compounds in the active sites of the proteins were made. The other selected compounds were predicted to have the same trend since they were all small molecules with small molecular weight. All the compounds bind with minimal energies all registering negative energies. This meant that the compounds were binding with ease. This clearly shows that the compounds are active against Plasmodium falciparum.

In the tables above which shows the results of docked complex between the ligand and the protein, the solution no shows the ranking of various complexes made when the ligand is in various positions in the molecule. The score refers to the geometric shape complementary score. Each of the solution is ranked according to this score with the highest being solution no 1 . The area in the table refers to the interface area of the complex. ACE refers to the atomic contact energy. This is the energy the ligand uses to fit in the protein. The solution with the minimal energy was ranked high as it meant that the ligand fitted perfectly well without much strain. Transformation is the 3D transformation. This is the 3 rotational angles and 3 translational parameters of the ligand molecule in the complex formed.

\section{Appendices}

\begin{tabular}{|c|c|}
\hline Swiss Model & $\begin{array}{r}\text { This is a scientific online tool used in converting the PDB sequence of the two proteins into 3D } \\
\text { structures. }\end{array}$ \\
\hline Open Babel & $\begin{array}{r}\text { This is a chemical tool which can interpret different languages and commands. It is used to } \\
\text { convert the smiles of the compounds into a 3D coordinate structure. }\end{array}$ \\
\hline Pymol & This tool is used to view the generated 3D structure. The PDB file is opened and viewed through \\
\hline
\end{tabular}




\section{Open Access Journal of Pharmaceutical Research}

\begin{tabular}{|c|c|}
\hline $\begin{array}{c}\text { Schrödinger's } \\
\text { suite }\end{array}$ & $\begin{array}{c}\text { this tool. } \\
\text { and the protein structures. After different poses of the ligand in the protein structure is } \\
\text { generated, they are ranked and the best pose is ranked first. }\end{array}$ \\
\hline Patch Dock & $\begin{array}{c}\text { Tool used in the docking experiment. This tool is used to bind the compound and the protein } \\
\text { structures. After different poses of the ligand in the protein structure is generated, they are } \\
\text { ranked and }\end{array}$ \\
\hline
\end{tabular}

Table 6: Various tools and software suit used during the course of the research with details of what each software suit was used for

The protein sequences of the templates:

40S ribosomal protein S7 [Plasmodium falciparum] MDAVQKRVLKSNPSDLEKEIAQCLLDIELSSSSDIKTDAKEIK LLSCDLIEVEKLKKKTILIYIPYKIYTTYVRKIQRKLINELEKKT KKYVVLVAKRTILKGKQKNKSFKIIPRSRTLTSVYDSILEDIVS PSEIIGKRISMKADGKRVFKIMLDSKERQRDNIEEKLISFAAV YKKITRRDAVFSLPPTNEK

40S ribosomal protein S19 [Plasmodium falciparum] MAEQFTEDIGVVNKRLLEPVPFVKTNNCIKDVDADLFIRSYA THLKLHNKITYPKWCTFVKTGKGRKLAPLNEDWYFIRASSIL RRLYLHPDIGVGFLRRQFSSKQRRGVAPNHTSLASGKILRSIL QQLENLGYVEQNPKKKGRRLTTKGENAINNFARYINKKVYN KE

\section{Conclusion}

The experiment was successfully carried out the objectives of the study were all achieved. The 3D structures for the selected 20 compounds were obtained and they were found to perfectly dock in the in the two proteins S7 and S19. The compounds can hence be synthesized as antiplasmodial drugs after in vitro studies are done. The following recommendations should be done: (a) the docking process should be done for all the compounds in the Pathogen Box. (b) The compounds found to have bind perfectly well in the proteins used, should be modified by adding some active functional groups to the compounds and docked again to check if their activities against Plasmodium falciparum has improved. (c) These compounds can eventually be synthesized in the lab and their antiplasmodial activities tested towards drug development.

\section{Acknowledgment}

A global team supported this work and we'd thank Dr. Fred Sijenyi for initial contributions to this project and DNA SOFTWARE Inc for RNA123 access, to Dr. Muge and Maureen Michire for their invaluable help in the manuscript revision and editions. Further support by Medicines for Malaria Venture (MMV), and the Chinese Government for the travel fellowships to Institute Materia Medica Symposium of drug discovery of infectious diseases, May 2015. Part of this research was conducted at University of Nairobi School of computing and informatics.

\section{References}

1. Nissen P, Hansen J, Ban N, Moore PB, Steitz TA (2000) The structural basis of ribosome activity in peptide bond synthesis. science 289(5481): 920-930.

2. Ferreira-Cerca S, Pöll G, Gleizes PE, Tschochner H, Milkereit P (2005) Roles of eukaryotic ribosomal proteins in maturation and transport of pre-18S rRNA and ribosome function. Mol cell 20(2): 263-275.

3. O'Donohue MF, Choesmel V, Faubladier M, Fichant G, Gleizes PE (2010) Functional dichotomy of ribosomal proteins during the synthesis of mammalian $40 \mathrm{~S}$ ribosomal subunits. J Cell Biol 190(5): 853-866.

4. Ibidapo CA (2005) Perception of causes of malaria and treatment-seeking behaviour of nursing mothers in a rural community. Aust J Rural Health 13(4): 214218. 


\section{Open Access Journal of Pharmaceutical Research}

5. Bonven B, Gulløv K (1979) Peptide chain elongation rate and ribosomal activity in Saccharomyces cerevisiae as a function of the growth rate. Mol Gen Genet 170(2): 225-230.

6. Oakes M, Henderson E, Scheinman A, Clark M, Lake JA (1986) Ribosome structure, function, and evolution: Mapping ribosomal RNA, proteins, and functional sites in three dimensions. Structure, function, and genetics of ribosomes Pp: 47-67.

7. Barlow R (1967) The economic effects of malaria eradication. The American Economic Review 57(2): 130-148.

8. Arjen M Dondorp, François Nosten, Poravuth Yi, Debashish Das, Aung Phae Phyo, et al. (2009) Artemisinin resistance in Plasmodium falciparum malaria. N Engl J Med 361(5): 455-467.

9. Wernsdorfer WH (1994) Epidemiology of drug resistance in malaria. Acta Trop 56(2): 143-156.

10. Mathenge P, Mwangi HNU, Mulaa F, Wagacha P, Sijenyi $F$ (2015) In silico structure of the 40s ribosomal subunit from plasmodium falciparum as drug targetby homology and de novo modeling.
11. Krogstad DJ, Gluzman IY, Kyle DE, Oduola AM, Martin SK, et al. (1987) Eflux of chloroquine from Plasmodium falciparum: mechanism of chloroquine resistance. Science 238(4831): 1283-1285.

12. Mwangi HNU, Wagacha P, Mathenge P, Sijenyi F, Mulaa $F$ (2018) Integrating mechanism-based screening paradigm into homology and de novo modeling exemplified by Mycobacterium Tuberculosis 30S ribosomal structure and its potential application as a screening target. International Journal of Scientific \& Engineering Research 9(2): 305-317.

13. Mwangi HNU (2013) Structure of the 40S ribosomal subunit from Plasmodium falciparum By Homology and De novo modeling (Doctoral dissertation, University of Nairobi).

14. Organization WH (2010) Global report on antimalarial drug efficacy and drug resistance: 20002010. Pp: 121.

15. Mwangi HNU, Wagacha P, Mathenge P, Sijenyi F, Mulaa F (2017) Structure of the 40S ribosomal subunit of Plasmodium falciparum by homology and de novo modeling. Acta pharmaceutica sinica B 7(1): 97-105. 